\title{
Serum IL-1 $\beta$ can be a biomarker in children with severe persistent allergic rhinitis
}

\author{
Myung Woul Han ${ }^{1 *} \mathbb{D}$, Song Hee Kim', Inbo Oh², Yang Ho Kim³ and Jiho Lee ${ }^{3 *}$
}

\begin{abstract}
Background: Allergic rhinitis (AR) is one of the most common diseases globally and usually persists throughout life. In the present study, we aimed to determine whether the expression of inflammatory biomarkers has a relationship with the severity of allergic rhinitis and with comorbid asthma or other allergic diseases in children.

Methods: For diagnosis of AR, the skin prick test was performed to measure the responses to 18 allergens. Blood levels of eosinophils and immunoglobulin $\mathrm{E}(\mathrm{lgE})$ were examined. We classified the patients into 2 groups based on the severity of the condition as Group 1 [intermittent AR (IAR) or mild persistent AR (PAR)] and Group 2 (moderate to severe PAR). To determine the expression of inflammatory biomarkers, in serum and several biomarkers (caspase-1, IL-1 $\beta, C C L-11, C C L-24$ and IL-33) were measured in the serum using enzyme-linked immunosorbent assay (ELISA). Additionally, we analyzed the correlation between clinical variables and the expression of biomarkers (eosinophils count, IL-1 $\beta$ and $C(L-24)$ and the severity of AR.
\end{abstract}

Results: We found that eosinophils count, IL-1 $\beta$, a marker of activation of inflammasomes, and CCL-24 were significantly increased in the moderate to severe PAR group ( $p=0.008, p=0.003, p=0.039$ ). Additionally, the expressions of eosinophil count, IL-1 $\beta$ and CCL-24 were significantly higher in patients with active asthmatic symptoms than in those without these conditions. On univariate analysis, allergic rhinitis in sibling, paternal allergic rhinitis, high expression of eosinophils count, IL-1 $\beta$ and CCL-24, history of active asthma and atopy correlated with severity of AR. Multivariate analysis showed only paternal allergic rhinitis and high expression of IL-1 $\beta$ as significant risk factors of moderate to severe PAR with 6.4 fold and 4.7 fold-increase in risk, respectively ( $p=0.011$ and $p=0.030$ ).

Conclusion: In conclusion, this study provides the first evidence that an excessive release of biologically active IL-1 $\beta$ may promote inflammation in severe PAR. It demonstrates that IL-1 $\beta$ can be a biomarker for active allergic diseases such as AR, asthma, and atopy. Moreover, this finding suggests that IL-1B should be investigated as a therapeutic target in severe PAR and other allergic diseases.

Keywords: Allergic rhinitis, Asthma, Atopy, IL-1 $\beta$, Biomarker

\section{Introduction}

Allergic rhinitis (AR) is one of the most common diseases worldwide and usually persists throughout life. The prevalence of self-reported AR is estimated to be approximately $2 \%$ to $25 \%$ in children. AR is frequently

\footnotetext{
*Correspondence: brightmoon@uuh.ulsan.kr; leejh@uuh.ulsan.kr

${ }^{1}$ Department of Otolaryngology, Ulsan University Hospital, University

of Ulsan College of Medicine, 877 Bangeojinsunhwan-doro, Dong-gu, Ulsan 44033, Republic of Korea

${ }^{3}$ Department of Occupational and Environmental Medicine, Ulsan University Hospital, University of Ulsan College of Medicine, 877

Bangeojinsunhwan-doro, Dong-gu, Ulsan 44033, Republic of Korea Full list of author information is available at the end of the article
}

associated with asthma. Compared to other medical conditions, AR might not appear to be serious because it is not associated with severe morbidity or mortality. However, AR reduces the quality of life of many patients by impairing sleep quality and cognitive function and causing irritability and fatigue. It is associated with decreased school and work performance, especially during the peak pollen season [1-3]. In addition, uncontrolled moderate-to-severe AR affects asthma control $[4,5]$ and AR itself can be a risk factor for uncontrolled asthma in children [6]. According to the allergic rhinitis and its impact on asthma (ARIA) 
classification, AR is subdivided into intermittent allergic rhinitis (IAR) and persistent allergic rhinitis (PAR). The severity of allergic rhinitis can be classified as mild or moderate-severe depending on the severity of the symptoms and their impact on social life, and school and work performance [3, 7].

The signs and symptoms of AR are the result of an IgE-mediated allergic reaction involving different cells, mediators, cytokines, chemokines, neuropeptides, and other components of a complex immunological network [8]. Various biomarkers can be obtained by several more or less non-invasive sampling methods to evaluate the nasal allergic response and the disease activity in AR [8-10]. While there are a lot of studies about diagnostic biomarkers that help identify patients with allergic rhinitis, limited studies have been conducted on prognostic biomarkers that are of value in evaluating the risk of disease progression or severity of allergic rhinitis $[8,11-14]$. Assessment of inflammation or prediction of severity using biomarkers offers a more objective and direct read-out that can contribute to our understanding of the mechanisms of allergic rhinitis, helps monitor disease severity, and is useful in evaluating the effects of (novel) treatments [8]. A large number of studies investigating various biomarkers for AR have been published over the past decades regarding their diagnostic and/or predictive value $[8,15-17]$.

Inflammatory biomarkers can aid in establishing the diagnosis, in staging and monitoring of the disease activity/progression, or in predicting or monitoring of a treatment response. Especially in (young) children, reliable, non-invasive biomarkers would be valuable. Cytokine levels and other mediators have been investigated in patients with rhinitis using the nasal fluid $[8,9,18]$, either to get more insight regarding the pathogenic condition, for diagnostic purposes, or to measure therapeutic effects. However, no single or specific biomarker for AR has been identified to date, especially in children's serum, for predicting the severity of the condition $[9,18,19]$.

Inflammasomes are key inflammatory signaling platforms that detect microbial substances, sterile environmental insults, and molecules derived from host cells. Activation of inflammasomes promotes caspase1-mediated secretion of proinflammatory cytokines such as interleukin (IL)-1 $\beta$ and IL-18 and pyroptosis. It is likely that excessive inflammasome activation and IL- $1 \beta$ responses contribute to the complex inflammatory processes in the development of COPD and neutrophilic asthma as well as infection-associated exacerbations that promote progression to more severe disease $[20,21]$. IL- $1 \beta$ is a key molecule and its release plays a crucial role in the pathogenesis of allergic asthma [22]. Thus, antagonizing the function of IL- $1 \beta$ holds promise as a potential therapeutic option for the treatment of asthma [20-23].

In the present study, we aimed to determine whether the expression of inflammatory biomarkers has a relationship with the severity of allergic rhinitis and with comorbid asthma or other allergic diseases in children. Moreover, we also tried to analyze the impact of environmental factors on PAR.

\section{Patients and methods Study subjects}

Subjects included 3097 children enrolled in the Elementary School Students Cohort (2015-2016) for identifying environmental factors of allergic disease in the Environmental Health Center of Ulsan University Hospital (Ulsan, Korea). The study participants were recruited from 4 elementary schools in Ulsan. Parental informed consent was obtained before enrollment. This study was approved by the institutional ethics review committee of Ulsan University Hospital (approval number 2009-09-061-011). At the time of enrollment, the subjects were given a detailed questionnaire and laboratory tests were performed. All the students responded to the questionnaire and underwent routine medical checkups, including a skin prick test and a blood test for total IgE levels and eosinophil counts. The questionnaires used in this study consisted of the International Society of Asthma and Allergy of Children (ISAAC) survey questions [24], and questions regarding the patients' socioeconomic status and hazardous environmental factors. The parents or legal guardians of all participants provided written informed consent. We assembled a cohort of patients diagnosed with AR to undertake a nested case-control study. We identified all children who were diagnosed as having allergic rhinitis based on clinical symptoms and objective tests of IgEmediated skin prick test.

\section{Diagnosis of allergic rhinitis}

We used the clinical definition of AR for diagnosis, namely, symptoms of allergic rhinitis including rhinorrhea, nasal obstruction, nasal itching, and sneezing, which are reversible spontaneously or with treatment, and objective tests of IgE-mediated allergy, such as the skin prick test [2]. As part of the study, the skin prick test was performed during a routine medical examination, to measure the responses to 18 allergens, which were compared to a positive and a negative control. Positive reactions for each allergen were defined as a wheal diameter at least $3 \mathrm{~mm}$ greater than that of the negative control. 
Measurement and analysis of serum cytokine, chemokine, and inflammasome levels

Blood levels of eosinophils and immunoglobulin $\mathrm{E}$ (IgE) were examined. To determine the expression of inflammatory biomarkers in patients with AR, several biomarkers were measured in the serum. The expression of interleukin (IL)-33 [25] (an epithelial biomarker that plays an important role in the development of allergic diseases), $\mathrm{C}-\mathrm{C}$ motif chemokine ligand (CCL)-11, CCL-24 [26, 27] (chemokines that induce eosinophils in allergic diseases), and caspase- 1 and IL- $1 \beta$ (which represent the activation of inflammasomes) $[20,21]$ were measured by enzyme-linked immunosorbent assay (ELISA). The details of each of the ELISA kits used in the study are as follows: Human CCL24/Eotaxin-2 ELISA (catalog no. DCC240B), CCL11/Eotaxin ELISA (catalog No. DTX00), IL-33 ELISA (catalog No. D3300), and caspase-1/ICE-1ß/IL-1F2 (catalog No. DLB50). The kits were obtained from R \& D Systems (Minneapolis, MN).

\section{Statistical analysis}

The independent $t$ test and the $\mathrm{x}^{2}$ test were used to analyze baseline differences. Univariate and multivariate analyses were performed to determine possible relationships between the variables and the development of severe AR. Multiple logistic regression analyses were performed using variables found to be significant $(P<0.05)$ in the univariate analysis and those reported to be associated with the development of severe AR. A $P$ value less than 0.05 was considered statistically significant in all analyses. Analyses of the receiver operating characteristic (ROC) curve and the differences in the area under curves (AUC) were used to estimate the diagnostic accuracy of each test.

\section{Results}

\section{Patient characteristics}

We selected 116 children who had experienced AR in the 1 year and in whom the severity of symptoms and duration could be classified according to the ARIA classification. Table 1 presents the characteristics of study participants. The mean age was 8.66 years (range, 6-12 years) and boys and girls were 69 and 47, respectively.

The mean eosinophil (\%), eosinophils count and IgE levels were $4.55 \pm 2.54 \%, 316.41 \pm 187.72$ count/ $\mu \mathrm{l}$ and $441.65 \pm 929.42 \mathrm{IU} / \mathrm{ml}$, respectively. And the mean caspase-1, IL-1 $\beta$, CCL-11, CCL-24, and IL-33 levels were $97.70 \pm 46.33 \mathrm{pg} / \mathrm{ml}, \quad 12.45 \pm 13.29 \mathrm{pg} /$ $\mathrm{ml}, 30.34 \pm 30.35 \mathrm{pg} / \mathrm{ml}, 482.53 \pm 301.81 \mathrm{pg} / \mathrm{ml}$, and $0.75 \pm 4.15 \mathrm{pg} / \mathrm{ml}$, respectively.

We classified the patients into 2 groups based on the duration of symptoms and severity of the condition as follows: Group 1 (intermittent allergic rhinitis, IAR and mild persistent allergic rhinitis, PAR) and Group 2 (moderate to severe persistent allergic rhinitis, PAR). There were 79 patients in Group 1 and 37 patients in Group 2.

\section{Comparison of inflammatory biomarkers}

At first, we analyzed the eosinophil and IgE level, markers of allergy in each group (Table 2 and Fig. 1). When we compared the levels of the markers, we found that

Table 1 Characteristics of children with allergic rhinitis $(N=116)$

\begin{tabular}{|c|c|}
\hline Demographic factors & \\
\hline Age & $\begin{array}{l}8.66 \pm 1.67 \text { years } \\
\text { (range, } \\
6-12 \text { years) }\end{array}$ \\
\hline Boys/girls & $69 / 47$ \\
\hline Height (cm) & $134.05 \pm 11.20$ \\
\hline Weight (kg) & $31.58 \pm 8.58$ \\
\hline Laboratory factors & \\
\hline Eosinophil (\%) & $4.55 \pm 2.54$ \\
\hline Eosinophils (count/ $\mu$ l) & $316.74 \pm 187.72$ \\
\hline Total lgE (IU/ml) & $441.65 \pm 929.42$ \\
\hline Caspase (pg/ml) & $97.70 \pm 46.33$ \\
\hline $\mid \mathrm{L}-1 \beta(\mathrm{pg} / \mathrm{ml})$ & $12.45 \pm 13.29$ \\
\hline CCL-11 (pg/ml) & $84.69 \pm 30.35$ \\
\hline CCL-24 (pg/ml) & $482.53 \pm 301.81$ \\
\hline IL-33 (pg/ml) & $0.75 \pm 4.15$ \\
\hline
\end{tabular}


eosinophils count in group 2 was significantly higher than group 1 ( $p=0.008)$. But, total IgE level was no significant difference between two groups. And eosinophils count was significantly higher in AR with active asthma than AR without active symptoms ( $305.9 \pm 176.9$ vs $557.1 \pm 277.3$, $p=0.003$ ) but, there was no significant difference when it comes to asthma history and atopy (Fig. 1).

Next, we investigated the expression of other inflammatory markers, and in group 1 , the mean caspase-1, IL-1 $\beta$, CCL-11, CCL-24, and IL-33 levels were $96.59,9.98,82.84,443.04$, and 0.50 , respectively. In group 2, the mean caspase-1, IL-1 $\beta$, CCL-11, CCL-24, and IL-33 levels were 100.08, 17.72, 88.63, 566.83, and 1.32 , respectively. When we compared the levels of the inflammatory biomarkers, we found that IL- $1 \beta$, a marker of activation of inflammasomes, and CCL-24, a marker of activation of eosinophils, were significantly increased in Group 2 ( $p=0.003, p=0.039$, Fig. 2a).

Additionally, we investigated the difference in expression of biomarkers between patients with AR and asthma $(\mathrm{N}=23)$ and those with $A R$ alone $(\mathrm{N}=93)$. In terms of differences in the expression of biomarkers between patients with AR and asthma and patients with AR alone, we found that in the group with asthma, expression of CCL-24 were significantly higher than in the group without asthma $(p=0.006$, Fig. $2 b)$. And the expression of IL-1 $\beta$ and CCL-24 in patients with activation of asthma $(\mathrm{N}=9)$ in 1 year was significantly higher than in children without activation of asthma $(p<0.001$ and $p=0.004$, Fig. 2c). In children with active atopic symptoms (skin rash) within 1 year $(\mathrm{N}=37)$, the expression of IL-1 $\beta$ was significantly higher than in children without atopic symptoms $(p=0.049$, Fig. 2d). In Group 2, there was no significant difference of eosinophils count, IL-1 $\beta$ and CCL-24 when the expression was compared between patients with AR and asthma $(\mathrm{N}=10)$ and patients with $A R$ alone $(\mathrm{N}=27$, data was not shown). Collectively, these results suggest that eosinophils count, IL-1 $\beta$ and CCL-24 were overexpressed in children with moderate to severe PAR and with other active allergic diseases and therefore, can be used as a biomarker of exacerbation or activation of allergic diseases including AR. Additionally, we investigated correlation between eosinophil count and IL-1 $\beta$ and

Table 2 The comparison of blood eosinophil and total $\lg E$ level according to severity of allergic rhinitis

\begin{tabular}{lccc}
\hline & Group 1 & Group 2 & P value \\
\hline Eosinophil (\%) & $4.19 \pm 2.56$ & $5.073 \pm 2.23$ & 0.075 \\
Eosinophils (count/Ml) & $285.61 \pm 178.72$ & $383.34 \pm 191.65$ & 0.008 \\
Total lgE (IU/ml) & $376.52 \pm 837.01$ & $571.25 \pm 909.92$ & 0.258 \\
\hline
\end{tabular}

CCL-24 expression which overexpression was shown in severe AR and the significant correlation was shown between IL-1 $\beta$ and CCL-24 $(p=0.0042)$, but there was no significant correlation between eosinophils count and IL-1 $\beta$ or eosinophils count and CCL-24 $(p=0.8123$, $p=0.180)$.

\section{Independent risk factors for the development of moderate to severe PAR}

We also tried to determine whether eosinophils count, IL-1 $\beta$ and CCL-24 expression could be used diagnostically in patients with moderate to severe PAR with classification according to the cut-off value of IL-1 $\beta$ and CCL-24 using ROC curves. We analyzed the correlation between clinical variables including perinatal factors, environmental factors, parental and socioeconomic factors, family history of allergic disease, and activation of other allergic symptoms and the severity of AR. For eosinophils count, with a cut-off value of 285.2 count/ $\mu \mathrm{l}$, there were 79 children with low expression and 37 children with high expression using ROC. For IL-1 $\beta$, with a cut-off value of $7.98 \mathrm{pg} / \mathrm{ml}$, there were 64 children with low expression and 32 children with high expression using ROC. For CCL-24, with a cut-off value of $471.01 \mathrm{pg} / \mathrm{ml}$, there were 56 children with low expression and 60 children with high expression using ROC. Table 3 presents the results of the univariate analysis of factors with regard to the presence of moderate to severe PAR. On univariate analysis, history of AR in sibling, history of paternal AR, high eosinophils count $(\geq 285.2)$, high expression of IL-1 $\beta$ ( $\geq 7.98 \mathrm{pg} / \mathrm{ml})$, CCL-24 ( $\geq 471.01)$, history of active asthma symptoms within 1 year and active atopic symptoms within 1 year, were associated with the development of moderate to severe PAR $(p=0.039, p=0.002, p=0.029, p=0.002, p=0.017$, $p=0.003$, and $p=0.050$, respectively). Perinatal factors, environmental factors, and socioeconomic factors did not show correlation with the development of moderate and severe PAR. After multivariate analysis, only paternal allergic rhinitis (6.4-fold increase in risk) and high expression of IL-1 $\beta$ (4.7-fold increase in risk), emerged as significant risk factors of moderate to severe PAR ( $p=0.011$ and $p=0.030$, respectively, Table 4 ).

\section{Discussion}

Inflammatory biomarkers can aid in establishing the diagnosis, in staging and monitoring of the disease activity/progression, or in predicting or monitoring of a treatment response. Especially in (young) children, reliable, non-invasive biomarkers would be valuable. Cytokine levels and other mediators have been investigated in patients with rhinitis using the nasal fluid $[8,9,18]$, either to get more insight regarding the 
a

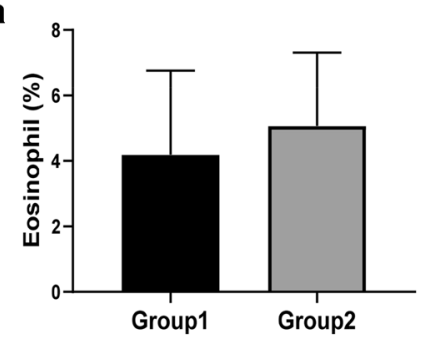

b

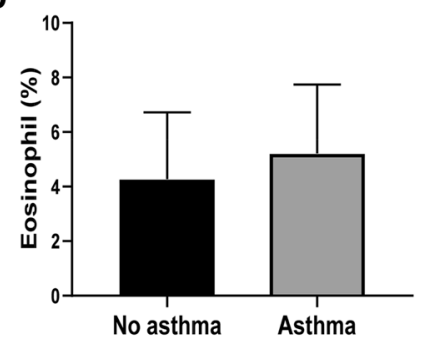

C

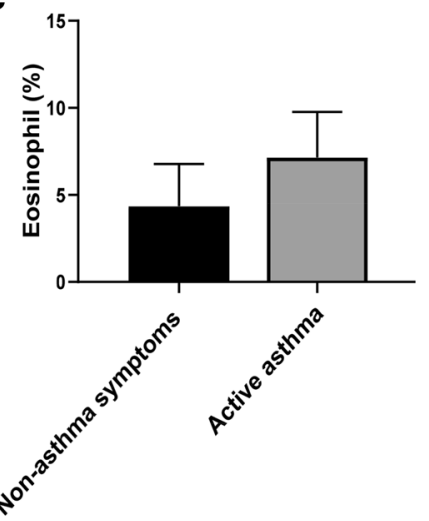

d

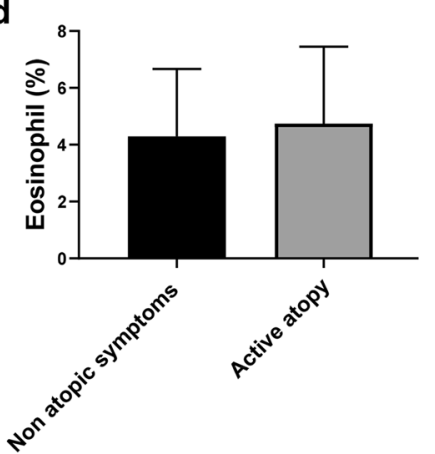

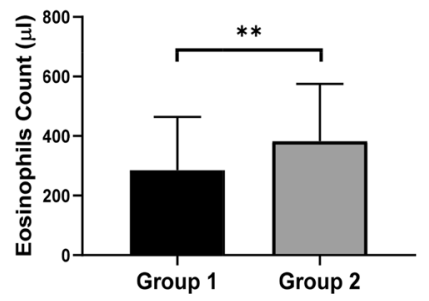
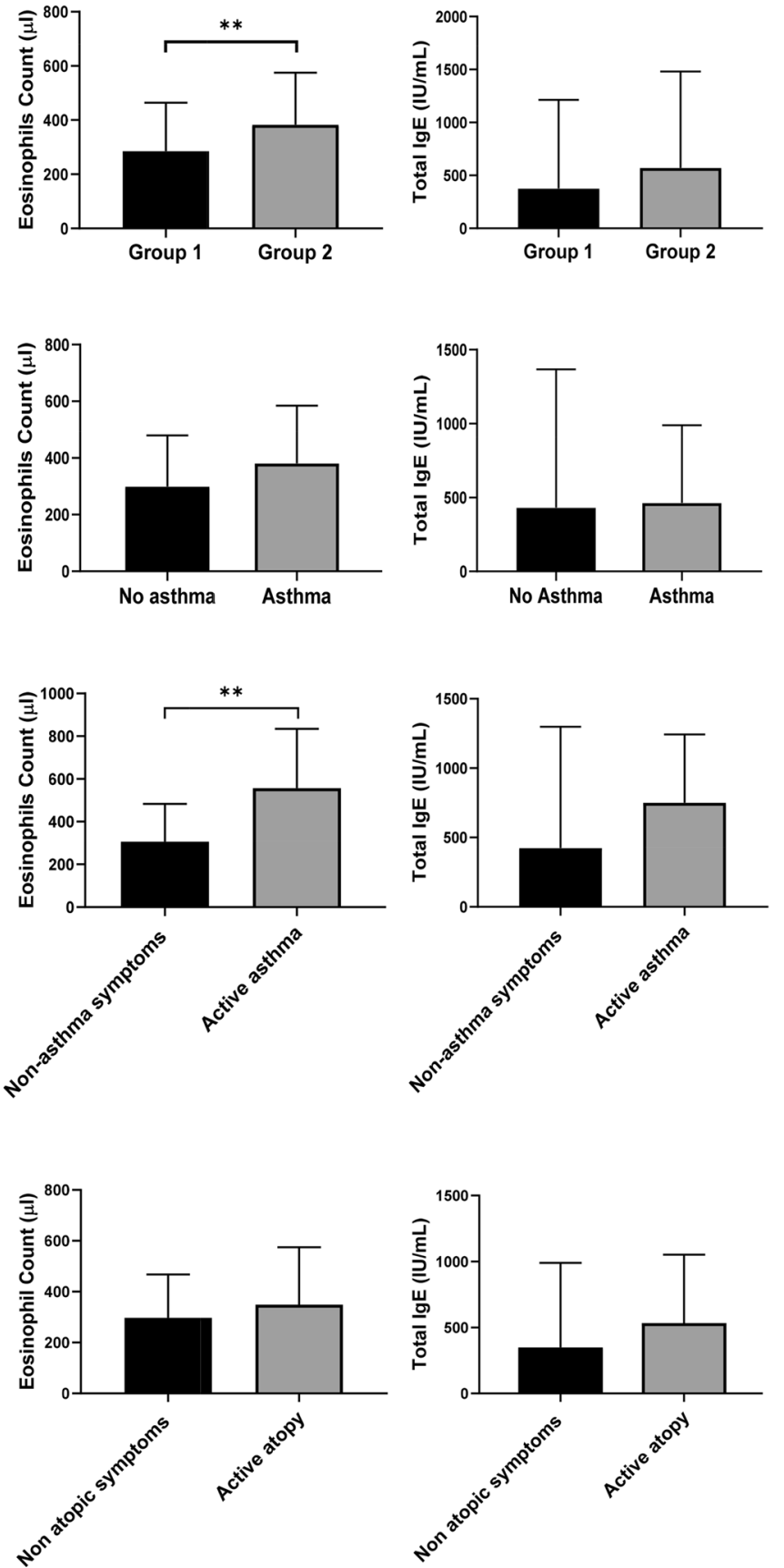

Fig. 1 Comparison of the mean concentrations of eosinophil (\%), eosinophil count and total IgE of blood in the IAR or mild PAR group (Group 1) and the moderate to severe PAR group (Group 2). According to severity of rhinitis (a), asthma history (b), active symptom of asthma (c), active symptom of atopy $(\mathbf{d}) .{ }^{*} \mathrm{P}<0.05 .{ }^{* * P}<0.01,{ }^{* * *} \mathrm{P}<0.001$

pathogenic condition, for diagnostic purposes, or to measure therapeutic effects. However, no single or specific biomarker for AR has been identified to date, especially in children's serum, for predicting the severity of the condition $[9,18,19]$. In children, measurements of inflammatory markers are inconsistent across the different (sampling) techniques reflecting disease heterogeneity, methodological limitations, or varying sensitivity of the biomarker detection techniques [9, $12,18,19]$. Hence, at this stage, biomarkers cannot be 

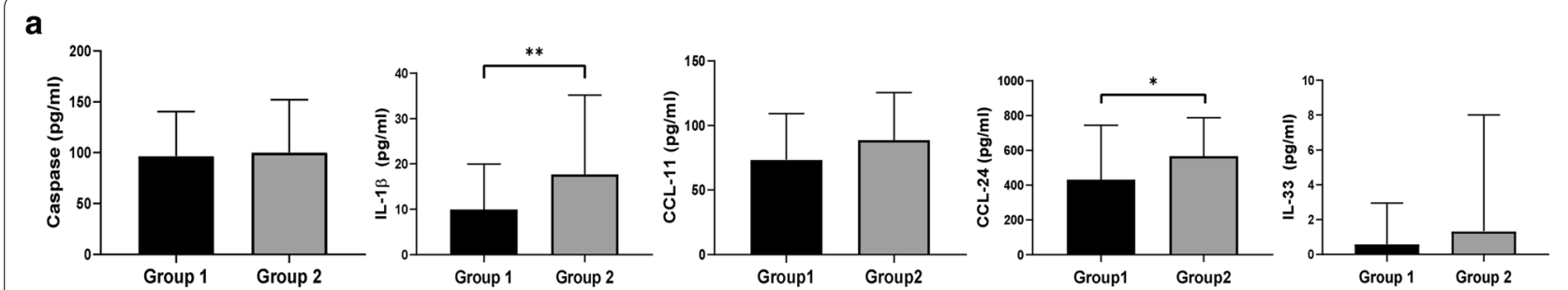

b
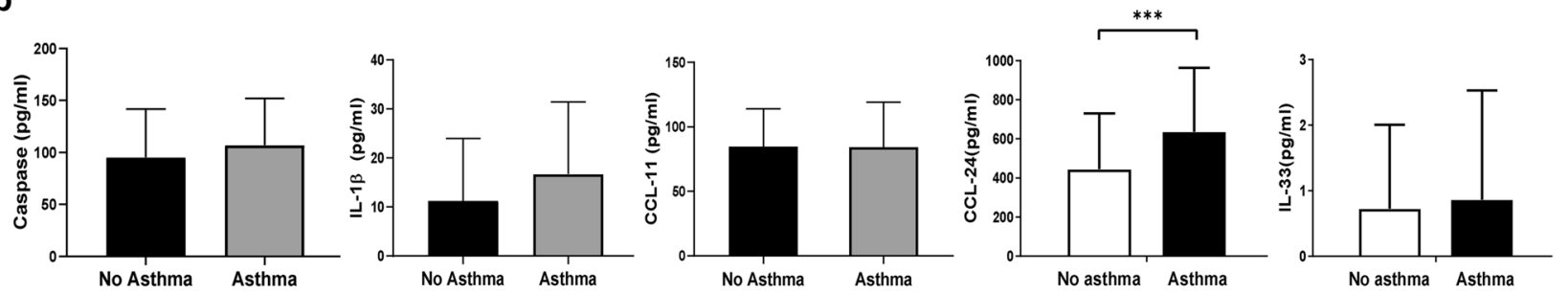

C
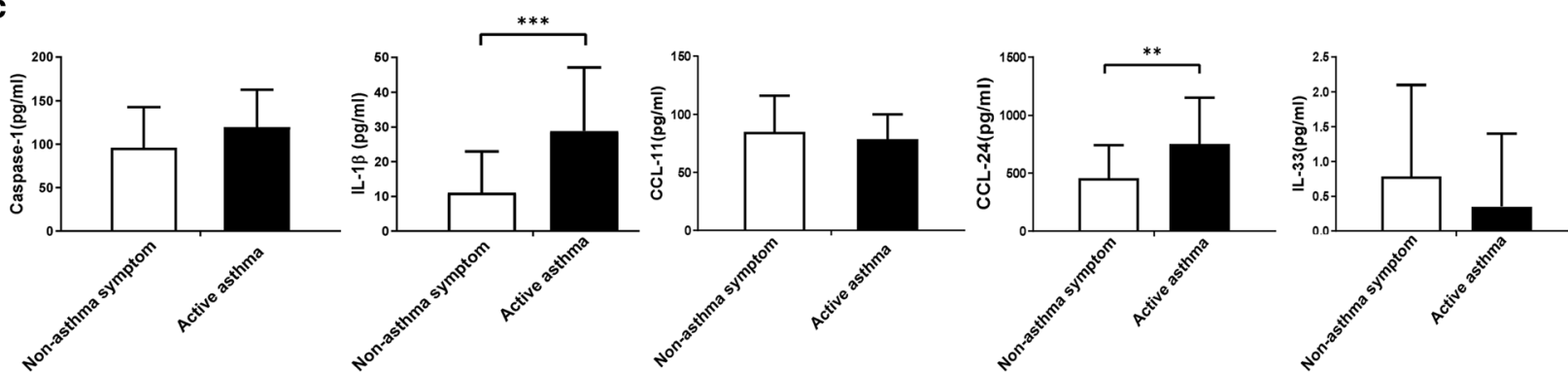

d
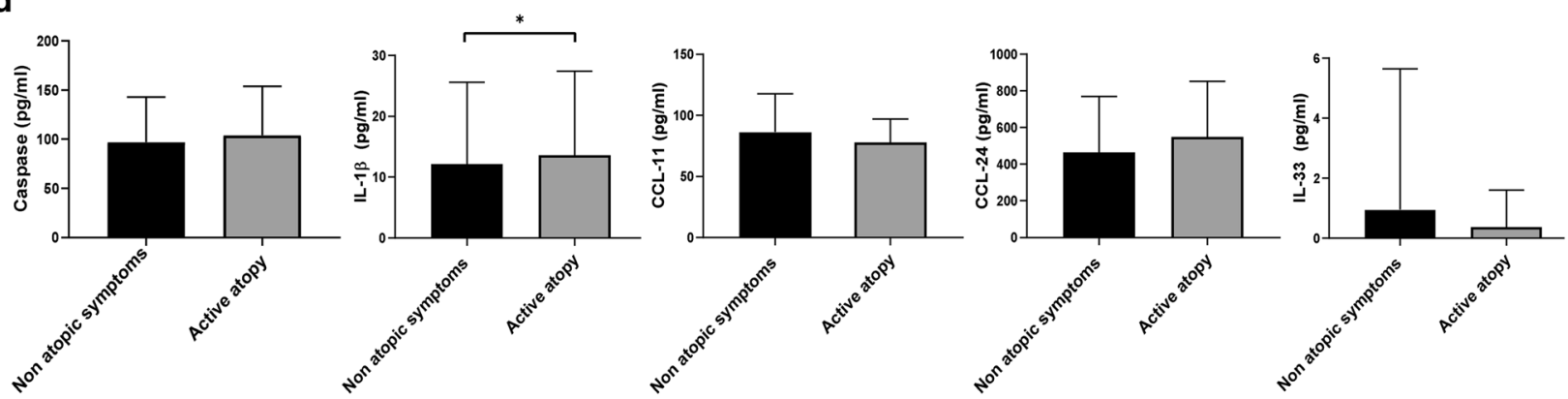

Fig. 2 Comparison of the mean concentrations of caspase-1, IL-1 B, CCL-11, CCL-24, and IL-33 a in the IAR or mild PAR group (Group 1) and the moderate to severe PAR group (Group 2), $\mathbf{b}$ in children with and without asthma, $\mathbf{c}$ in children with and without active symptoms of asthma within 1 year and $\mathbf{d}$ in children with and without active symptoms of atopy (skin rash) within 1 year. ${ }^{*} P<0.05$. ${ }^{*} P<0.01,{ }^{* * *} P<0.001$. CCL: $C-C$ motif chemokine ligand; IL: interleukin

generally recommended as reliable tools to evaluate or treat a child with AR. The research and development of biomarkers using relatively non-invasive techniques, such as using blood and urine samples, will help predict the severity and prognosis in AR and asthma patients and will contribute to the development of new therapeutic targets.

There are no data at present regarding the role of IL- $1 \beta$ in AR in children. Our nested case-control study demonstrated that elevated IL- $1 \beta$ levels and paternal allergic rhinitis have a significant effect on the severity of AR. To the best of our knowledge, this is the first study to compile and evaluate the expression of inflammasomerelated proteins in children with AR using a serum sample. The current study had an adequate sample size and used strict inclusion criteria to ensure that a well-characterized group of subjects with moderate to severe PAR (according to ARIA guidelines) could 
Table 3 Univariate analysis to determine factors related to the severity of allergic rhinitis

\begin{tabular}{llll}
\hline AR severity & $\begin{array}{l}\text { Group 1 } \\
(\mathrm{N}=79)\end{array}$ & $\begin{array}{l}\text { Group 2 } \\
(\mathrm{N}=37)\end{array}$ & $p$ value \\
& & \\
\hline
\end{tabular}

Perinatal factors

Delivery

Delivery by cesarean section $\quad 33 \quad 12$

Birth weight

LBW $(<2.5 \mathrm{~kg})$

$\mathrm{NBW} \geq 2.5 \mathrm{~kg}$

Prematurity

Prematurity $(<37$ weeks) $\quad 8 \quad 3$

Full-term ( $\geq 37$ weeks) $\quad 71$

Oxygen therapy in the first week

$$
\text { No }
$$

Yes

Breastfeeding

No

Yes

General anesthesia before 1 year

No

Yes

Environmental factors

Tobacco smoking

No
Yes

Distance to major roadway or factory

$$
<100 \mathrm{~m} \quad 53
$$$$
\geq 100 \mathrm{~m}
$$

Exposure to traffic exhaust

$$
\text { No }
$$$$
\text { Yes }
$$

Family history of allergy

Allergic disease in sibling

$$
\text { No }
$$$$
\text { Yes }
$$

Allergic rhinitis in sibling

$$
\text { No }
$$$$
\text { Yes }
$$

Paternal allergic disease

$$
\text { No }
$$$$
\text { Yes }
$$

Paternal allergic rhinitis

$$
\text { No }
$$$$
\text { Yes }
$$

Maternal allergic disease

$$
\text { No }
$$$$
\text { Yes }
$$

Maternal allergic rhinitis

$$
\text { No }
$$$$
\text { Yes }
$$

0.415

25

2

3

34

32

5

9

28

36

1

32

4

24

13

24

3

0.920

0.331

14

17

25

60

21

10

0.176

15

55

23

15

22

\begin{tabular}{|c|c|c|c|}
\hline AR severity & $\begin{array}{l}\text { Group } 1 \\
(\mathrm{~N}=79)\end{array}$ & $\begin{array}{l}\text { Group } 2 \\
(\mathrm{~N}=37)\end{array}$ & $p$ value \\
\hline \multicolumn{4}{|c|}{ Activation of other allergic symptoms (history in 1 year) } \\
\hline \multicolumn{4}{|c|}{ Active asthma } \\
\hline No & 76 & 31 & 0.029 \\
\hline Yes & 3 & 6 & \\
\hline \multicolumn{4}{|l|}{ Active atopic symptoms } \\
\hline No & 62 & 17 & 0.002 \\
\hline Yes & 18 & 19 & \\
\hline \multicolumn{4}{|l|}{ Active allergic conjunctivitis } \\
\hline No & 57 & 24 & 0.516 \\
\hline Yes & 22 & 13 & \\
\hline \multicolumn{4}{|l|}{ History of asthma } \\
\hline No & 66 & 27 & 0.215 \\
\hline Yes & 13 & 10 & \\
\hline \multicolumn{4}{|l|}{ Eosinophils (count/ $\mu \mathrm{l})$} \\
\hline Low expression $(<285.2)$ & 45 & 34 & 0.017 \\
\hline High expression ( $\geq 285.2)$ & 12 & 25 & \\
\hline \multicolumn{4}{|l|}{$\mathrm{IL}-1 \beta(\mathrm{pg} / \mathrm{ml})$} \\
\hline Low expression $(<7.98)$ & 51 & 13 & 0.003 \\
\hline High expression ( $\geq 7.98)$ & 28 & 24 & \\
\hline \multicolumn{4}{|l|}{$\mathrm{CCL}-24(\mathrm{pg} / \mathrm{ml})$} \\
\hline Low expression $(<471.01)$ & 43 & 13 & 0.050 \\
\hline High expression ( $\geq 471.01)$ & 36 & 24 & \\
\hline
\end{tabular}

62

14

26

5

48

16

20
Table 3 (continued)

Group 1: Children with intermittent allergic rhinitis or mild persistent allergic rhinitis; Group 2: children with moderate to severe persistent allergic rhinitis AR Allergic rhinitis, CCL C-C motif chemokine ligand, IL interleukin, LBW low birth weight, $N B W$ normal birth weight

Table 4 Multivariate analysis to determine factors related to the severity of the persistent allergic rhinitis

\begin{tabular}{lllll}
\hline & OR & P value & 95\% Cl \\
\hline Allergic rhinitis in sibling & 1.098 & 0.295 & 0.151 & 1.774 \\
Paternal allergic rhinitis & 6.426 & 0.011 & 0.086 & 0.731 \\
Eosinophil count $(<285.2$ vs $\geq 285.2)$ & 2.833 & 0.092 & 0.143 & 1.159 \\
IL-1 $\beta(<7.98$ vs $\geq 7.98)$ & 4.697 & 0.030 & 0.122 & 0.900 \\
$C C L-24(<471.01$ vs $\geq 471.01)$ & 1.233 & 0.267 & 0.205 & 5.376 \\
Active atopy & 2.028 & 0.154 & 0.093 & 1.366 \\
Active asthma & 4.070 & 0.253 & 0.367 & 45.138 \\
\hline CCL C-C motif chemokine ligand, Cl confidence interval, IL interleukin, OR odds \\
ratio
\end{tabular}

be observed alongside a group of mild PAR subjects. Basically, we investigated the blood IgE and eosinophil level, the marker of allergic response. It is known that nasal eosinophils and basophils result in the late-phase allergic response. Nasal smear eosinophil counts is highly specific criterion for the diagnosis of AR but are poor indicators of the degree, duration, or type of upper or 
associated lower airway inflammation due to allergy [28]. High blood eosinophil count is a risk factor for incident asthma and eosinophilic inflammation of the airways characterises disease severity in subsets of individuals with severe asthma and there is a direct relationship between eosinophil count and the frequency of asthma exacerbation $[29,30]$. It was unknown about the role of blood eosinophils count in AR and we demonstrated that the higher eosinophils count can be biomarker of severity or activation of allergic disease although it was not significant risk factor for severe PAR on multivariate analysis.

In the present study, we also investigated the chemokine ligand levels, that is, CCL11 (also known as eotaxin), and CCL24 (also known as eotaxin-2 or myeloid progenitor inhibitor factor-2), which are all epithelial cell-derived chemokines involved in the pathogenesis of asthma and rhinitis. It is known that CCL5, CCL11, and CCL24 may contribute to the airway recruitment of fibrocytes in severe asthma [26]. CCL24 is likely involved in the pathogenesis of chronic nasal hypereosinophilia; nasal fluid CCL24 levels show a significant correlation with the degree of eosinophilia and clinical symptoms [31, 32]. In our study, CCL-24 was significantly overexpressed in severe PAR and in children with asthma, although it did not show up as a significant risk factor for severe PAR on multivariate analysis. These results suggest that CCL-24 may contribute to airway inflammation in severe allergic rhinitis and active symptomatic asthma. We found the correlation between IL-1 $\beta$ and CC- 24 and these biomarkers may play important role in development of inflammation in AR.

The current study demonstrated that IL- $1 \beta$ was strong risk factor to develop of moderate to severe PAR and other active allergic diseases, and therefore, it can be a biomarker of exacerbation or activation of allergic diseases, including AR. The primary role of the inflammasomes is to direct inflammation by controlling unregulated IL- $1 \beta$ release; therefore, IL- $1 \beta$ can be a significant marker of activation of allergic disease or severity. Recent literature has indicated that IL-1 $\beta$ levels are increased in bronchoalveolar lavage fluid and sputum of asthmatic patients compared with those seen in healthy volunteers, and IL-1 blockade can be a novel target for asthma therapy. IL-1ra is a natural anti-inflammatory cytokine that competes with IL-1 $\beta$ for binding to the IL- 1 receptor, preventing IL- $1 \beta$ from binding to the IL-1 type I receptor and effectively reduced airway inflammation [33]. Therefore, new therapeutic approaches for the treatment of allergic diseases, such as asthma and severe rhinitis, could be aided by the development of agents that target the IL-1 $\beta$.

In this study, paternal but not maternal allergic rhinitis had a significant effect on the severity of AR. We cannot guess the reason and meanings accurately, but there is chance that results from higher portion of boys. Recent article indicates a sex dependent association of parental allergic conditions with childhood allergies; maternal allergy increasing the risk in girls and paternal allergy in boys [34]. There were some limitations to our study. First, this study had geographic selection bias, Ulsan of Korea and may not be generalized to all children or adults and second, we only determined expression of inflammatory biomarkers at once.

To date, no single or specific biomarker for allergy, especially for severe PAR, has been identified [9, 18, 35]. As allergy is not one disease, but a collection of a number of allergic conditions, it is therefore not very plausible that one marker would fit all, and therefore, a more holistic approach using a combination of clinical history, clinical readouts, and diagnostic markers will be needed for correct diagnosis.

\section{Conclusion}

In conclusion, this study provides the first evidence for the hypothesis that an excessive release of biologically active IL-1 $\beta$ may promote inflammation in severe PAR. It also demonstrates that IL- $1 \beta$ can be a biomarker of active allergic diseases such as AR, asthma, and atopy. Moreover, this finding suggests that IL-1B should be investigated as a therapeutic target in severe PAR and other allergic diseases. Further studies will help fully elucidate the potential role of IL-1 $\beta$ in AR treatment. Moreover, identification of accurate biomarkers in patients who are unlikely to respond to conventional therapy may promote the development of rational drug combinations that will overcome this problem.

\section{Abbreviations}

IAR: intermittent allergic rhinitis; PAR: persistent allergic rhinitis; OR: odds ratios; $\mathrm{Cl}$ : confidence intervals; CCL: C-C motif chemokine ligand; IL: interleukin; LBW: low birth weight; NBW: normal birth weight.

\section{Authors' contributions}

$\mathrm{MH}$ and $J \mathrm{~L}$ designed the study. MH, SK, IO, YK and $J \mathrm{~L}$ coordinated the overall undertaking of the study. $\mathrm{MH}$ and $J \mathrm{~L}$ performed statistical analyses. $\mathrm{MH}$ and $\mathrm{J}$ wrote the manuscript. All authors contributed to the revision. All authors read and approved the final manuscript.

\section{Funding}

This work received funding from a Grant from the Environmental Health Center funded by the Ministry of Environment, Republic of Korea and a Grant from the Korea Health Technology R\&D Project through the Korea Health Industry Development Institute (KHIDI), funded by the Ministry of Health \&Welfare, Republic of Korea (Grant HC15C1335).

\section{Availability of data and materials}

The datasets used and/or analyzed during the current study are available from the corresponding author on reasonable request.

\section{Ethics approval and consent to participate}

This study was approved by the institutional ethics review committee of Ulsan University Hospital (approval number 2009-09-061-011). The parents or legal guardians of all participants provided written informed consent. 


\section{Consent for publication}

Not applicable.

\section{Competing interests}

The authors declare that they have no competing interests.

\begin{abstract}
Author details
1 Department of Otolaryngology, Ulsan University Hospital, University of Ulsan College of Medicine, 877 Bangeojinsunhwan-doro, Dong-gu, Ulsan 44033, Republic of Korea. ${ }^{2}$ Environmental Health Center, University of Ulsan College of Medicine, Ulsan, Republic of Korea. ${ }^{3}$ Department of Occupational and Environmental Medicine, Ulsan University Hospital, University of Ulsan College of Medicine, 877 Bangeojinsunhwan-doro, Dong-gu, Ulsan 44033 , Republic of Korea.
\end{abstract}

Received: 8 January 2019 Accepted: 27 August 2019 Published online: 18 September 2019

\section{References}

1. Brozek JL, Bousquet J, Baena-Cagnani CE, Bonini S, Canonica GW, Casale TB, van Wijk RG, Ohta K, Zuberbier T, Schunemann HJ. Allergic rhinitis and its impact on asthma (ARIA) guidelines: 2010 revision. J Allergy Clin Immunol. 2010;126:466-76.

2. Bousquet J, Khaltaev N, Cruz AA, Denburg J, Fokkens WJ, Togias A, Zuberbier T, Baena-Cagnani CE, Canonica GW, van Weel C, et al. Allergic rhinitis and its impact on asthma (ARIA) 2008 update (in collaboration with the World Health Organization, GA(2)LEN and AllerGen). Allergy. 2008;63(Suppl 86):8-160.

3. Jauregui I, Davila I, Sastre J, Bartra J, del Cuvillo A, Ferrer M, Montoro J, Mullol J, Molina X, Valero A. Validation of ARIA (allergic rhinitis and its impact on asthma) classification in a pediatric population: the PEDRIAL study. Pediatr Allergy Immunol. 2011;22:388-92.

4. Corren J, Adinoff AD, Buchmeier AD, Irvin CG. Nasal beclomethasone prevents the seasonal increase in bronchial responsiveness in patients with allergic rhinitis and asthma. J Allergy Clin Immunol. 1992;90:250-6.

5. Taramarcaz P, Gibson PG. Intranasal corticosteroids for asthma control in people with coexisting asthma and rhinitis. Cochrane Database Syst Rev. 2003. https://doi.org/10.1002/14651858.CD003570.

6. Xiang L, Zhao J, Zheng Y, Liu H, Hong J, Bao Y, Chen A, Deng L, Ji W, Zhong N, Shen K. Uncontrolled asthma and its risk factors in Chinese children: a cross-sectional observational study. J Asthma. 2016;53:699-706.

7. Brozek JL, Bousquet J, Agache I, Agarwal A, Bachert C, Bosnic-Anticevich S, Brignardello-Petersen R, Canonica GW, Casale T, Chavannes NH, et al. Allergic rhinitis and its impact on asthma (ARIA) guidelines-2016 revision. J Allergy Clin Immunol. 2017;140:950-8.

8. Diamant Z, Boot JD, Mantzouranis E, Flohr R, Sterk PJ, Gerth van Wijk R. Biomarkers in asthma and allergic rhinitis. Pulm Pharmacol Ther 2010;23:468-81.

9. Knipping K, Knippels LM, Dupont C, Garssen J. Serum biomarkers for allergy in children. Pediatr Allergy Immunol. 2017;28:114-23.

10. Colavita L, Catalano N, Sposito G, Loddo S, Galletti B, Salpietro C, Galletti F, Cuppari C. Local allergic rhinitis in pediatric patients: is IgE dosage in nasal lavage fluid a useful diagnostic method in children? Int J Mol Cell Med. 2017;6:174-82.

11. Ciprandi G, Gallo F, Ricciardolo FL, Cirillo I. Fractional exhaled nitric oxide: a potential biomarker in allergic rhinitis? Int Arch Allergy Immunol. 2017;172:99-105.

12. Zicari AM, Occasi F, Di Fraia M, Mainiero F, Porzia A, Galandrini R, Giuffrida A, Bosco D, Bertin S, Duse M. Local allergic rhinitis in children: novel diagnostic features and potential biomarkers. Am J Rhinol Allergy. 2016;30:329-34.

13. Hsu PY, Yang YH, Lin YT, Chiang BL. Serum eosinophil cationic protein level and disease activity in childhood rhinitis. Asian Pac J Allergy Immunol. 2004;22:19-24.

14. Chen ST, Sun HL, Lu KH, Lue KH, Chou MC. Correlation of immunoglobulin $\mathrm{E}$, eosinophil cationic protein, and eosinophil count with the severity of childhood perennial allergic rhinitis. J Microbiol Immunol Infect. 2006;39:212-8.
15. Quek YW, Sun HL, Ng YY, Lee HS, Yang SF, Ku MS, Lu KH, Sheu JN, Lue KH. Associations of serum leptin with atopic asthma and allergic rhinitis in children. Am J Rhinol Allergy. 2010;24:354-8.

16. Fitzpatrick AM. Biomarkers of asthma and allergic airway diseases. Ann Allergy Asthma Immunol. 2015;115:335-40.

17. Durham SR, Di Cara G, Marcucci F, Palomba A, Milioni M, Pecoraro L, Ciprandi G, Buttafava S, Frati F, Verrotti A. Exhaled nitric oxide in children with allergic rhinitis: a potential biomarker of asthma development. Am J Respir Crit Care Med. 2015;26:85-7.

18. Hofmaier S. Allergic airway diseases in childhood: an update. Pediatr Allergy Immunol. 2014;25:810-6.

19. James A, Hedlin G. Biomarkers for the phenotyping and monitoring of asthma in children. Clin Exp Allergy. 2016;3:439-52.

20. Kim RY, Pinkerton JW, Gibson PG, Cooper MA, Horvat JC, Hansbro PM. Inflammasomes in COPD and neutrophilic asthma. Thorax. 2015:70:1199-201.

21. Howrylak JA, Nakahira K. Inflammasomes: key mediators of lung immunity. Annu Rev Physiol. 2017:79:471-94.

22. Ritter M, Straubinger K, Schmidt S, Busch DH, Hagner S, Garn H, Prazeres da Costa C, Layland LE. Functional relevance of NLRP3 inflammasomemediated interleukin (IL)-1 beta during acute allergic airway inflammation. Clin Exp Immunol. 2014;178:212-23.

23. Hoffman HM, Wanderer AA. Inflammasome and IL-1 beta-mediated disorders. Curr Allergy Asthma Rep. 2010;10:229-35.

24. Kim A, Lim G, Oh I, Kim Y, Lee T, Lee J. Perinatal factors and the development of childhood asthma. Ann Allergy Asthma Immunol. 2018;120:292-9.

25. Ohno T, Morita H, Arae K, Matsumoto K, Nakae S. Interleukin-33 in allergy. Allergy. 2012;67:1203-14

26. Isgro M, Bianchetti L, Marini MA, Bellini A, Schmidt M, Mattoli S. The C-C motif chemokine ligands CCL5, CCL11, and CCL24 induce the migration of circulating fibrocytes from patients with severe asthma. Mucosal Immunol. 2013;6:718-27.

27. Dai C, Yao X, Gordon EM, Barochia A, Cuento RA, Kaler M, Meyer KS, Keeran KJ, Nugent GZ, Jeffries KR, et al. A CCL24-dependent pathway augments eosinophilic airway inflammation in house dust mitechallenged Cd163(-/-) mice. Mucosal Immunol. 2016;9:702-17.

28. Pal I, Sinha Babu A, Halder I, Kumar S. Nasal smear eosinophils and allergic rhinitis. Ear Nose Throat J. 2017;96:E17-e22.

29. Matucci A, Vultaggio A, Maggi E, Kasujee I. Is IgE or eosinophils the key player in allergic asthma pathogenesis? Are we asking the right question? Respir Res. 2018;19:113.

30. Bai C, Jiang D, Wang L, Xue F, Chen O. A high blood eosinophil count may be a risk factor for incident asthma in population at risk. Respir Med. 2019;151:59-65.

31. De Corso E, Baroni S, Battista M, Romanello M, Penitente R, Di Nardo W, Passali GC, Sergi B, Fetoni AR, Bussu F, et al. Nasal fluid release of eotaxin-3 and eotaxin-2 in persistent sinonasal eosinophilic inflammation. Int Forum Allergy Rhinol. 2014;4:617-24.

32. De Corso E, Baroni S, Romitelli F, Luca L, Di Nardo W, Passali GC, Paludetti G. Nasal lavage CCL24 levels correlate with eosinophils trafficking and symptoms in chronic sino-nasal eosinophilic inflammation. Rhinology. 2011:49:174-9.

33. Hernandez ML, Mills K, Almond M, Todoric K, Aleman MM, Zhang H, Zhou H, Peden DB. IL-1 receptor antagonist reduces endotoxin-induced airway inflammation in healthy volunteers. J Allergy Clin Immunol. 2015;135:379-85.

34. Arshad SH, Karmaus W, Raza A, Kurukulaaratchy RJ, Matthews SM, Holloway JW, Sadeghnejad A, Zhang H, Roberts G, Ewart SL. The effect of parental allergy on childhood allergic diseases depends on the sex of the child. J Allergy Clin Immunol. 2012;130(427-434):e426.

35. Eifan AO, Orban NT, Jacobson MR. Severe Persistent Allergic Rhinitis. Inflammation but no histologic features of structural upper airway remodeling. Am J Respir Crit Care Med. 2015:192:1431-9.

\section{Publisher's Note}

Springer Nature remains neutral with regard to jurisdictional claims in published maps and institutional affiliations. 